TRANSACTIONS OF THE

AMERICAN MATHEMATICAL SOCIETY

Volume 348, Number 11, November 1996

\title{
THE DYNAMICAL PROPERTIES OF PENROSE TILINGS
}

\author{
E. ARTHUR ROBINSON, JR.
}

\begin{abstract}
The set of Penrose tilings, when provided with a natural compact metric topology, becomes a strictly ergodic dynamical system under the action of $\mathbf{R}^{2}$ by translation. We show that this action is an almost 1:1 extension of a minimal $\mathbf{R}^{2}$ action by rotations on $\mathbf{T}^{4}$, i.e., it is an $\mathbf{R}^{2}$ generalization of a Sturmian dynamical system. We also show that the inflation mapping is an almost 1:1 extension of a hyperbolic automorphism on $\mathbf{T}^{4}$. The local topological structure of the set of Penrose tilings is described, and some generalizations are discussed.
\end{abstract}

\section{INTRODUCTION}

Penrose tilings are a remarkable family of aperiodic tilings of the plane, first described in the mid 1970s by Roger Penrose [11]. They consist of the edge-toedge tilings of $\mathbf{R}^{2}$ by copies of the two marked Penrose tiles (Figure 1, on the next page), subject to the matching rule that the arrows on adjacent edges must match (Figure 2).

While it is not completely trivial to show that any Penrose tilings exist, there are in fact uncountably many incongruent Penrose tilings [7], all of which are aperiodic. It has often been noted that many of the basic properties of Penrose tilings are similar to properties well known in dynamical systems theory. For example, the matching rule resembles a subshift of finite type (the tiles play the role of the symbols and the arrows play the role of the adjacency graph). Similarly, the Penrose inflation property (see Section 7) resembles a substitution rule for a substitution dynamical system (cf. [3]). It has also been observed that the inflation is related to a certain hyperbolic toral automorphism (see [1] and [17]). In this paper we will make these and many other such analogies concrete by studying Penrose tilings in terms of tiling dynamical systems.

A tiling dynamical system consists of a translation invariant set of tilings with a natural compact metric topology on which $\mathbf{R}^{n}$ acts by translation. D. Rudolph [15] first introduced a particular tiling dynamical system as a device to generalize the idea of a 'flow under a function' (i.e., suspensions) to $\mathbf{R}^{n}$. More generally, tiling dynamical systems provide a natural notion of symbolic dynamics in the $\mathbf{R}^{n}$ setting. Our results in the Penrose tiling case concern both the ergodic theory and the topological dynamics of the Penrose tiling dynamical system. Our main result

Received by the editors May 13, 1995.

1991 Mathematics Subject Classification. Primary 28D05; Secondary 28D20.

Key words and phrases. Tilings, topological dynamics, almost periodicity.

Partially supported by a George Washington University Committee on Research UFF grant and by NSF grant DMS-9303498.

(C)1996 American Mathematical Society 

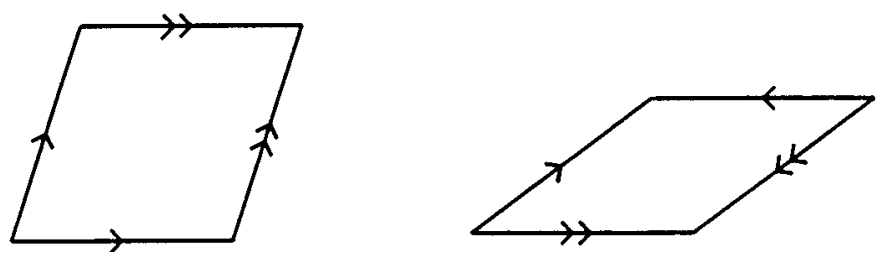

Figure 1. Penrose tiles.

is that this system is an almost 1:1 extension of a certain Kronecker $\mathbf{R}^{2}$ action on $\mathbf{T}^{4}$; the pure point spectrum of this Kronecker action is generated by the 5 th roots of unity. It follows that the Penrose system is strictly ergodic, with metric (but not topological) pure point spectrum. As a corollary, we obtain a simple description of the local topological structure of the compact metric space $X$ of all Penrose tilings. This should be compared to the more difficult $C^{*}$ algebra based 'topological structure' on the set of congruence classes of Penrose tilings recently discussed by A. Connes [4]. Finally, we show that the inflation mapping $Q$ on $X$ is an almost 1:1 extension of a hyperbolic automorphism of $\mathbf{T}^{4}$.

Our analysis of Penrose tilings is aided by the fact that an extensive understanding of Penrose tilings already exists. In particular, we make substantial use of N. G. de Bruijn's [2] remarkable algebraic structure theorem for Penrose tilings, although

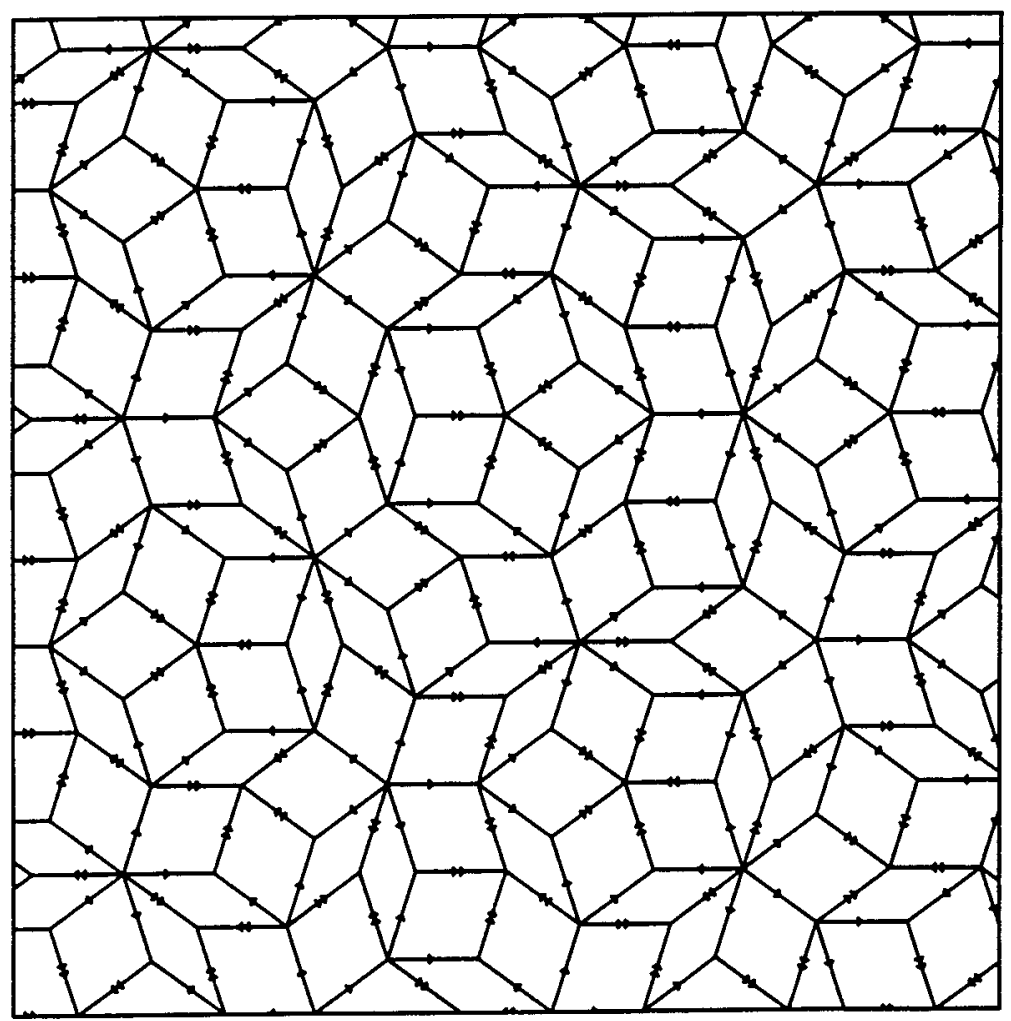

Figure 2. Part of a Penrose tiling of the plane. 
we need to work to make that result continuous and translation equivariant. This paper differs from previous approaches to Penrose tilings in that our results (and proofs) specifically involve dynamical ideas. For example, the proof of our main result shows that the Penrose tiling dynamical system is essentially a suspension of a $\mathbf{Z}^{2}$ symbolic dynamical system which is obtained as the product of two 1dimensional Sturmian systems. Our result on the topology of Penrose tiling space amounts to constructing $\mathbf{R}^{2}$ 'flow boxes' around arbitrary Penrose tilings. Other papers (see [13], [12] and [16]) have analyzed various types of aperiodic tilings in terms of tiling dynamical systems. However, the results presented here for the Penrose case are the most specific and complete results of this kind to date.

\section{TILING SPACES}

In this paper, a tile will be a convex polygon in $\mathbf{R}^{2}$ (possibly with markings). A tiling $x$ will be a set of tiles which cover $\mathbf{R}^{2}$, intersecting only on the boundaries. We will always regard two congruent tilings as different if they differ by a nontrivial rigid motion. For $t>0$, let $C_{t}=\left\{\left(v_{1}, \ldots, v_{n}\right):\left|v_{i}\right| \leq t\right\} \subseteq \mathbf{R}^{2}$. Given $\epsilon>0$ and a tiling $x$, let $\partial_{\epsilon}(x)=\partial\left(C_{1 / \epsilon}\right) \cup \bigcup_{\tau \in x}\left(\partial(\tau) \cap C_{1 / \epsilon}\right)$, where $\partial(\omega)$ is the boundary of $\omega$ (including the markings, if any). Let $N_{\epsilon}(\omega)=\bigcup_{\mathbf{v} \in \omega} B_{\epsilon}(\mathbf{v})$. For two compact subsets $\omega_{1}, \omega_{2} \subseteq \mathbf{R}^{n}$ let $H\left(\omega_{1}, \omega_{2}\right)=\max \left\{\inf \left\{\epsilon_{1}: \omega_{1} \subseteq N_{\epsilon_{1}}\left(\omega_{2}\right)\right\}, \inf \left\{\epsilon_{2}: \omega_{2} \subseteq N_{\epsilon_{2}}\left(\omega_{1}\right)\right\}\right\}$ be the Hausdorff metric. For tilings $x, y$, let $h(x, y)=\inf \left\{\epsilon: H\left(\partial_{\epsilon}(x), \partial_{\epsilon}(y)\right) \leq \epsilon\right\}$. One can show that $h$ defines a metric on any set $X$ of tilings of $\mathbf{R}^{2}$. Let $T^{\mathbf{t}} x$ denote the translation of $x$ by $\mathbf{t} \in \mathbf{R}^{2}$. We call a compact $T$-invariant set $X$ of tilings a shift space of tilings, and note that $(\mathbf{t}, x) \mapsto T^{\mathbf{t}}$ on $X$ is a continuous $\mathbf{R}^{2}$ action (i.e., a dynamical system) on $X$.

For a finite set $p$ of translationally incongruent prototiles, let $X_{p}$ denote the set of tilings of $\mathbf{R}^{2}$ by translations of tiles in $p$. If the tiles $p$ have matching-rule markings, we assume that $x \in X_{p}$ satisfies that rule. We always assume $X_{p} \neq \phi$. Rudolph [15] observed that $X_{p}$ is always compact (cf. [12]). Let $\tilde{p}$ be prototiles obtained by rotating the tiles in Figure 1 by multiples of $2 \pi / 10$, and let $p$ denote the tiles in $\tilde{p}$ without the markings. Then $\operatorname{card}(\tilde{p})=20, \operatorname{card}(p)=10$, and the $2: 1$ 'eraser' mapping $\varepsilon: \tilde{p} \rightarrow p$ extends to a continuous mapping $\varepsilon: \tilde{X} \rightarrow X_{p}$ satisfying $\varepsilon\left(T^{\mathbf{t}} x\right)=T^{\mathbf{t}} \varepsilon(x)$. We call $X=\varepsilon\left(X_{\tilde{p}}\right)$ the unmarked Penrose tilings. Since it is possible to restore the markings to a tile in a Penrose tiling knowing only its immediate neighbors (see [2]), $\varepsilon$ has a continuous inverse, and hence provides a topological conjugacy. ${ }^{1}$ Thus without loss of generality, we work with $X$.

In addition to being aperiodic, all Penrose tilings $x$ satisfy the local isomorphism property: for any patch $s$ of tiles in $x$, there exists $R>0$ such that a copy of $s$ occurs within $R$ from an arbitrary location in any Penrose tiling $y$ (including $y=x)$. Equivalently, in terms of toplogical dynamics, every Penrose tiling is an almost periodic point for $T$ (see [13]). By Gottschalk's Theorem [5], $T$ is minimal (i.e., there are no nontrivial closed $T$-invariant subsets of $X$ ).

\section{The KRONECKer Model}

For a continuous $\mathbf{R}^{2}$ action $T$ on a compact metric space $X$ we call $\mathbf{w} \in \mathbf{R}^{2}$ a continuous eigenvalue if there is a complex valued $f \in C(X)$ (called a continuous

\footnotetext{
${ }^{1}$ Similar topological conjugacies to other common kinds of Penrose tilings also exist, in particular: 'kites and darts', 'pentagons and stars', 'triangles' (cf. [7]).
} 
eigenfunction) such that

$$
f\left(T^{\mathbf{t}} x\right)=e^{2 \pi i<\mathbf{t}, \mathbf{w}>} f(x)
$$

for all $\mathbf{t} \in \mathbf{R}^{2}$. If $T$ is uniquely ergodic (i.e., there exists a unique $T$-invariant Borel probability measure $\mu$ on $X$ ), and (1) holds $\mu$ a.e. for a Borel function $f$ (i.e., a metric eigenfunction), we call $\mathbf{w}$ a metric eigenvalue. Let $C_{T}$ and $M_{T}$ (resp.) denote the continuous and metric eigenvalues. In the strictly ergodic (i.e., minimal and uniquely ergodic) case, both $C_{T}$ and $M_{T}$ are countable subgroups of $\mathbf{R}^{2}$ with $C_{T} \subseteq M_{T}$. We say $T$ has continuous (or metric) pure point spectrum if the span of the continuous (or metric) eigenfunctions is dense in $C(X)$ (or in $L^{2}(X, \mu)$ ). By the Halmos-von Neumann theorem, (see [19]) two minimal actions with topological pure point spectrum are topologically conjugate if and only if $C_{T}=C_{S}$. Such an action can always be realized as a Kronecker action, i.e., a group of rotations on a compact abelian group.

Let $\zeta=e^{2 \pi i / 5}$ and let $\mathbf{Z}[\zeta]$ denote the ring of integral combinations of the 5 th roots of unity. Since $1+\zeta+\zeta^{2}+\zeta^{3}+\zeta^{4}=0$ is the only integral relation among the 5 th roots of unity [8], the group $\mathbf{Z}[\zeta]$ is isomorphic to $\mathbf{Z}^{4}$, and the dual group $\widehat{\mathbf{Z}[\zeta]}$ is isomorphic to $\mathbf{T}^{4}=\mathbf{R}^{4} / \mathbf{Z}^{4}$. We 'decomplexify' $\mathbf{Z}[\zeta]$ and view it as the subset of $\mathbf{R}^{2}$ generated by the vectors $\left\{\mathbf{v}_{i}=\left(\operatorname{Re}\left(\zeta^{i}\right), \operatorname{Im}\left(\zeta^{i}\right)\right)\right\}$. We will model the Kronecker action $K$ of $\mathbf{R}^{2}$ on $\mathbf{T}^{4}$ with $C_{K}=M_{K}=\mathbf{Z}[\zeta]$ as follows: The set $\mathbf{T}_{0}^{5}=\left\{\mathbf{u} \in \mathbf{T}^{5}: u_{0}+\cdots+u_{4}=0(\bmod 1)\right\}$ is a closed subgroup of $\mathbf{T}^{5}$ isomorphic to $\mathbf{T}^{4}$. We identify $\mathbf{T}^{4}$ with a 4 -cube with opposite faces identified, and write the homeomorphism explicitly as $\pi\left(u_{0}, u_{1}, u_{2}, u_{4}\right)=\left(u_{0}, u_{1}, u_{2}, u_{3}, u_{4}\right)$, where $u_{3}=-\left(u_{0}+u_{1}+u_{2}+u_{4}\right)$. Let $W$ be the $5 \times 2$ matrix whose rows are $\mathbf{v}_{0}, \mathbf{v}_{1}, \ldots, \mathbf{v}_{4}$. Define a continuous action of $\mathbf{R}^{2}$ on $\mathbf{T}^{5}$ by $L^{\mathbf{t}} \mathbf{u}=\mathbf{u}+W \mathbf{t} \bmod 1$. Since $\mathbf{T}_{0}^{5}$ is $L$-invariant, we can define an action $K$ on $\mathbf{T}_{0}^{5}$ by $K^{\mathbf{t}}=\left.L^{\mathbf{t}}\right|_{\mathbf{T}_{0}^{5}}$. Pulling back by $\pi$ to $\mathbf{T}^{4}$, it is clear that $K$ is a strictly ergodic Kronecker action on $\mathbf{T}^{4}$ with $C_{K}=M_{K}=\mathbf{Z}[\zeta]$.

\section{The Penrose Shift}

Let $K$ and $T$ be $\mathbf{R}^{2}$ actions on compact metric spaces $Y$ and $X$ respectively. If $\varphi: X \rightarrow Y$ is a continuous surjection with $\varphi \circ T^{\mathbf{t}}=K^{\mathbf{t}} \circ \varphi$ then we say $K$ is a factor of $T$ ( $\varphi$ is the factor mapping) and $T$ is an extension of $K$. An extension is almost 1:1 if there exists $y \in Y$ such that $\operatorname{card}\left(\varphi^{-1}(y)\right)=1$. If $K$ is minimal then $\left\{y: \operatorname{card}\left(\varphi^{-1}(y)\right)=1\right\} \subseteq Y$ is a $K$-invariant and dense $G_{\delta}$ (see [18]). We define the thickness of the extension by $\eta=\sup \left\{\operatorname{card}\left(\varphi^{-1}(y)\right): y \in Y\right\}$, and we call $\left\{\operatorname{card}\left(\varphi^{-1}(y)\right): y \in Y\right\}$ the thickness spectrum. An extension is nontrivial if $\eta>1$. A nontrivial almost 1:1 extension of a strictly ergodic Kronecker action is called an almost automorphic action. Such actions are always minimal (see [18]).

Theorem A. The Penrose shift $T$ on $X$ is a strictly ergodic, almost automorphic action of $\mathbf{R}^{2}$ with thickness 10 (the thickness spectrum is $\{1,2,10\}$ ). The pure point spectrum factor is the Kronecker action $K$ on $\mathbf{T}^{4}$ with $C_{K}=\mathbf{Z}[\zeta]$, and the unique $T$-invariant measure on $X$ is the pullback $\mu=\lambda \circ \varphi^{-1}$ of Lebesgue measure $\lambda$ on $\mathbf{T}^{4}$.

It follows from Theorem A that $T$ has topological entropy zero. Geometrically this means the number of different 'pictures' occuring in $N \times N$ windows around the origin in all $x \in X$ grows sub-exponentially as a function of $N^{2}$. The one 
dimensional entropy in every direction is also zero. For fixed $\mathbf{t}_{0}$, let $T^{t \mathbf{t}_{0}}$ be the flow which translates each Penrose tiling in the direction $\mathbf{t}_{0}$. Theorem A implies that this flow is strictly ergodic if and only if $\left\{\mathbf{t}_{0}\right\}^{\perp} \cap \mathbf{Z}[\zeta]=\{0\}$. It follows that every possible patch occurs infinitely often in a sufficiently wide strip in such a direction $\mathbf{t}_{0}$. If $R_{\theta}$ denotes the rotation by $\theta$, then $T$ acting on $R_{\theta}(X)$ is topologically conjugate to $T$ acting on $X$ if and only if $\theta$ is a multiple of $2 \pi / 10$. Finally we note that $T$ on $X$ has metric pure point spectrum but not topological pure point spectrum since $\varphi$ is not 1:1. However, since $C_{T}=M_{T}$, every eigenfunction for $T$ is continuous, a property we refer to in [14] as homogeneity. The last statement is also proved in [16] by a completely different argument.

\section{The Proof of Theorem A}

For $u \in \mathbf{R}$, let $\ell_{j}(u)=\left\{\mathbf{t} \in \mathbf{R}^{2}:\left\langle\mathbf{t}, \mathbf{v}_{j}\right\rangle=u\right\}=\left\{\mathbf{v}_{j}\right\}^{\perp}+u \mathbf{v}_{j}$ in $\mathbf{R}^{2}$, and let $y_{j}(u)=\bigcup_{k \in \mathbf{Z}} \ell_{j}(u+k)$. Then $y_{j}$ is an infinite family of evenly spaced parallel lines, offset by $u$. We call $y_{j}(u)$ the $j$-grid and we call $\ell_{j}(u+k)$ the $k$-line in the $j$-grid. For $\mathbf{u} \in \mathbf{R}^{5}$ we let $y(\mathbf{u})=y_{0}\left(u_{0}\right) \cup y_{1}\left(u_{1}\right) \cup \cdots \cup y_{4}\left(u_{4}\right)$. We view $y(\mathbf{u})$ as a tiling of $\mathbf{R}^{2}$ by an infinite set of polygonal prototiles (see Figure 3 ), called a grid tiling.

Since $y(\mathbf{u}+\mathbf{k})=y(\mathbf{u})$ for $\mathbf{k} \in \mathbf{Z}^{5}$, we have $\mathbf{u} \in \mathbf{T}^{5}=\mathbf{R}^{5} / \mathbf{Z}^{5}$. For $\mathbf{u} \in \mathbf{R}^{5}$, $\mathbf{t} \in \mathbf{R}^{2}$, let $\mathbf{n}(\mathbf{t}, \mathbf{u})=\lfloor W \mathbf{t}+\mathbf{u}\rfloor \in \mathbf{Z}^{5}$ (where $\lfloor\cdot\rfloor$ denotes the 'floor'). Then $\mathbf{n}(\cdot, \mathbf{u}$ ) is constant on the tiles of $y(\mathbf{u})$, and changes by $\pm \mathbf{e}_{j}$ when a line in $y_{j}\left(u_{j}\right)$ is crossed. Let $W^{T}$ denote $W$ transpose, and define $b(\mathbf{u})=\left\{\frac{2}{5} W^{T} \mathbf{n}(\mathbf{t}, \mathbf{u}): \mathbf{t} \in \mathbf{R}^{2}\right\} \subseteq \mathbf{R}^{2}$.

For a tiling $y$, let $q(y)$ denote vertices of the tiles, and let $e(y)$ denote the edges. A tiling $x$ is dual to $y$ if there is a bijection $*: y \rightarrow q(x)$ such that when $\sigma_{1}, \sigma_{2} \in y$ are adjacent across an edge $\ell \in e(y)$, then the vertices $\sigma_{1}^{*}, \sigma_{2}^{*} \in q(x)$ are connected by the edge $\ell^{*} \in e(x)$. We call the duality $*$ geometric if each edge $\ell$ in $e(y)$ is perpendicular to $\ell^{*}$ in $e(x)$.

We call $y(\mathbf{u})$ nonsingular if no more than 2 grid lines cross at any point, and otherwise call it singular. We say $\mathbf{u} \in \mathbf{T}^{5}$ is nonsingular if $y(\mathbf{u})$ is nonsingular.

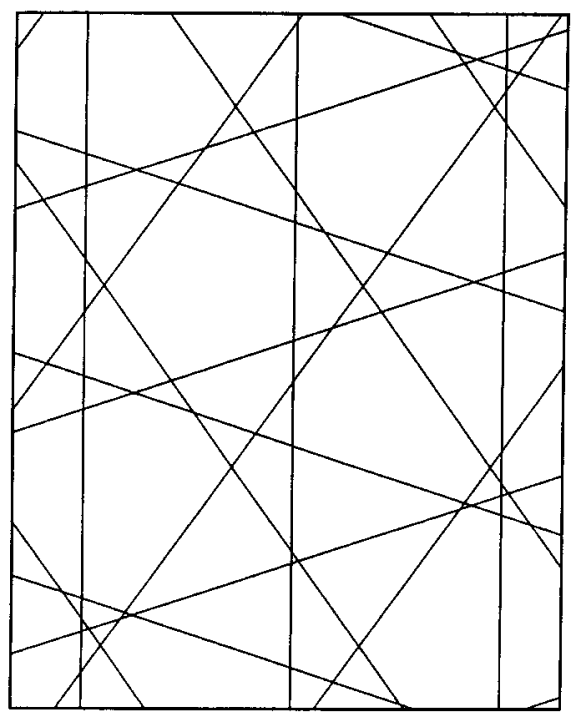

Figure 3. Part of a nonsingular grid tiling. 


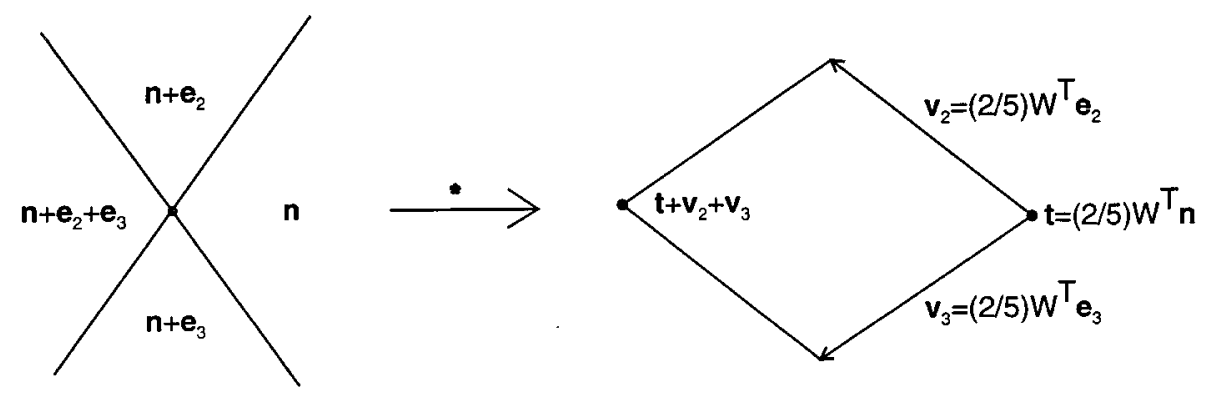

Figure 4. The Penrose tile dual to a vertex in a grid tiling.

We denote the nonsingular vectors by $\mathbf{T}_{n}^{5}$. Let $\mathbf{T}_{0, n}^{5}=\mathbf{T}_{0}^{5} \cap \mathbf{T}_{n}^{5}$ and note that $\mathbf{T}_{0, n}^{5} \subseteq \mathbf{T}_{0}^{5}$ is $K$-invariant and dense $G_{\delta}$. Let $\mathbf{R}_{n}^{5}$ be the lift of $\mathbf{T}_{n}^{5}$ to $\mathbf{R}^{5}$. We will use the following result.

Proposition 5.1 (de Bruijn [2]). Let $X_{p}$ denote the set of all edge-to-edge tilings by the unmarked Penrose prototiles $p$. For any $\mathbf{u} \in \mathbf{R}_{n}^{5}$ there exists $y^{*}(\mathbf{u}) \in X_{p}$ such that $q\left(y^{*}(\mathbf{u})\right)=b(\mathbf{u})$. In particular, for a tile $\sigma$ in $y(\mathbf{u})$ the vertex $\sigma^{*} \in b(\mathbf{u})$ in $y^{*}(\mathbf{u})$ is given by $\sigma^{*}=\frac{2}{5} W^{T} \mathbf{n}(\mathbf{t}, \mathbf{u})$ for any $\mathbf{t} \in \sigma$.

The main idea (see Figure 4) is that $\mathbf{v}_{j}=\frac{2}{5} W^{T} \mathbf{e}_{j}$ (see [2] for a proof).

The importance of the geometric property is that if $x, x^{\prime} \in X_{p}$ are both geometrically dual to $y(\mathbf{u})$, then $x^{\prime}=T^{\mathbf{t}} x$ for some $\mathbf{t} \in \mathbf{R}^{2}$. We will also use the following:

Theorem 5.2 (de Bruijn [2]). If $\mathbf{u} \in \mathbf{T}_{0, n}^{5}$ then $y^{*}(\mathbf{u})$ is a Penrose tiling.

Theorem 5.2 yields most Penrose tilings, up to translation. Unfortunately, it does not give all translations of the Penrose tilings it produces. Our next task is to correct this.

Let $\mathbf{m}(\mathbf{t}, \mathbf{u})=\mathbf{n}(\mathbf{t}, \mathbf{u})-\lfloor\mathbf{u}\rfloor=\lfloor W \mathbf{t}+\mathbf{u}\rfloor-\lfloor\mathbf{u}\rfloor$, so that ${ }^{2} \mathbf{m}(\mathbf{t}, \mathbf{u}+\mathbf{k})=\mathbf{m}(\mathbf{t}, \mathbf{u})$. Given $\mathbf{u} \in \mathbf{R}_{n}^{5}$, let

$$
\mathbf{t}(\mathbf{u})=\frac{2}{5} W^{T} \mathbf{u} \in \mathbf{R}^{2}
$$

and define

$$
x(\mathbf{u})=T^{\mathbf{t}(\mathbf{u})} y^{*}(\mathbf{u}) .
$$

If $q(\mathbf{u})=q(x(\mathbf{u}))$ is the vertex set, then $q(\mathbf{u})=b(\mathbf{u})-\mathbf{t}(\mathbf{u})=\frac{2}{5} W^{T}\{\mathbf{m}(\mathbf{t}, \mathbf{u})-\{\mathbf{u}\}$ : $\left.\mathbf{t} \in \mathbf{R}^{2}\right\}$, and thus $q(\mathbf{u}+\mathbf{k})=q(\mathbf{u})$. It follows that $q$ and $x$ depend on $\mathbf{u} \in \mathbf{T}_{0, n}^{5}$ $\left(\right.$ rather that $\mathbf{R}_{n}^{5}$ ). Let $X_{n}=\left\{x(\mathbf{u}): \mathbf{u} \in \mathbf{T}_{0, n}^{5}\right\}$. We call $x \in X_{n}$ a nonsingular Penrose tiling.

Lemma 5.3. The mapping $\mathbf{u} \mapsto x(\mathbf{u}): \mathbf{T}_{0, n}^{5} \rightarrow X_{n}$ is continuous and it satisfies $x\left(K^{\mathbf{t}} \mathbf{u}\right)=T^{\mathbf{t}} x(\mathbf{u})$.

Proof. Since $\mathbf{m}(\cdot, \mathbf{u})$ is constant on tiles in $y(\mathbf{u})$, a small change in $\mathbf{u}$ does not change $\mathbf{m}(\cdot, \mathbf{u})$ on a large square around $0 \in \mathbf{R}^{2}$.

\footnotetext{
${ }^{2}$ It is interesting to note that the function $\mathbf{m}$ satisfies a cocycle identity $\mathbf{m}(\mathbf{t}+\mathbf{s}, \mathbf{u})=$ $\mathbf{m}\left(\mathbf{t}, K^{\mathbf{s}} \mathbf{u}\right)+\mathbf{m}(\mathbf{s}, \mathbf{u})$.
} 
Since $\frac{2}{5} W^{T} W=I,(3)$ implies

$$
\begin{aligned}
q\left(K^{\mathbf{t}} \mathbf{u}\right) & =\frac{2}{5} W^{T}\left(\left\{\left\lfloor K^{\mathbf{t}} \mathbf{u}+W \mathbf{s}\right\rfloor: \mathbf{s} \in \mathbf{R}^{2}\right\}-(\mathbf{u}+W \mathbf{t})\right) \\
& =\frac{2}{5}\left(\left\{\lfloor\mathbf{u}+W(\mathbf{s}+\mathbf{t})\rfloor: \mathbf{s} \in \mathbf{R}^{2}\right\}-\mathbf{u}\right)-\mathbf{t} \\
& =\frac{2}{5} W^{T}\left(\left\{\lfloor\mathbf{u}+W \mathbf{s}\rfloor: \mathbf{s} \in \mathbf{R}^{2}\right\}-\mathbf{u}\right)-\mathbf{t} \\
& =q(\mathbf{u})-\mathbf{t} . \quad \square
\end{aligned}
$$

Lemma 5.4. There exists a uniformly continuous homeomorphism $\varphi: X_{n} \rightarrow \mathbf{T}_{0, n}^{5}$ such that $\mathbf{u}=\varphi(x(\mathbf{u}))$ and $\varphi\left(T^{\mathbf{t}} x\right)=K^{\mathbf{t}} \varphi(x)$.

The proof of Lemma 5.4 uses Sturmian dynamical systems ${ }^{3}$, which we briefly review. Let $\mathbf{T}=[0,1) \bmod 1$. For irrational $\alpha$, the rotation $R_{\alpha}$ on $\mathbf{T}$ (by $2 \pi \alpha$ ) is defined by $R_{\alpha} u=u+\alpha \bmod 1$. For $u \in \mathbf{T}$ define $z^{+}(u) \in\{0,1\}^{\mathbf{Z}}$ by $z^{+}(u)_{j}=0$ if $R_{\alpha}^{j} u \in[0, \alpha)$, and $z^{+}(u)_{j}=1$ if $R_{\alpha}^{j} u \in[\alpha, 1)$. Also define $z^{-}(u)_{j}=0$ if $R_{\alpha}^{j} u \in(0, \alpha]$, and $z^{-}(u)_{j}=1$ if $R_{\alpha}^{j} u \in(\alpha, 1]$. Let $Z^{+}=\left\{z^{+}(u): u \in \mathbf{T}\right\}, Z^{-}=\left\{z^{-}(u): u \in \mathbf{T}\right\}$, $Z=Z^{+} \cup Z^{-}$and $Z_{n}=Z^{+} \cap Z^{-}$. Then $Z$ is a closed shift-invariant subset of $\{0,1\}^{\mathbf{Z}}$. The set $Z \backslash Z_{n}$ is countable dense, and $Z_{n}$ is dense $G_{\delta}$. The Sturmian dynamical system is the left-shift $S$ acting on $Z$. We summarize some of its properties (see $[5])$.

Lemma 5.5. The shift $S$ on $Z$ is a thickness 2 almost automorphic extension of $R_{\alpha}$. The factor map $\psi: Z \rightarrow \mathbf{T}$ is $1: 1$ on $Z_{n}$ and $2: 1$ on $Z \backslash Z_{n}$. If $\mathbf{T}_{n}=\mathbf{T} \backslash\left\{R_{\alpha}^{n} 0\right.$ : $n \in \mathbf{Z}\}$, then $\left.\psi\right|_{Z_{n}}=\left(\left.z^{+}\right|_{\mathbf{T}_{n}}\right)^{-1}=\left(\left.z^{-}\right|_{\mathbf{T}_{n}}\right)^{-1}$.

Let $\Sigma\left(\mathbf{T}_{0}^{5}\right)=\left\{\mathbf{u} \in \mathbf{T}_{0}^{5}: u_{0}=u_{1}=0\right\}$ and $\Sigma\left(\mathbf{T}_{0, n}^{5}\right)=\Sigma\left(\mathbf{T}_{0}^{5}\right) \cap \mathbf{T}_{0, n}^{5}$. Let $w$ be the periodic tiling of $\mathbf{R}^{2}$ by the first two grids: $w=y_{0}(0) \cap y_{1}(0)$. The first two coordinates of $\left(\mathbf{m}_{0}(\mathbf{t}), \mathbf{m}_{1}(\mathbf{t})\right)$ of $\mathbf{m}(\mathbf{u}, \cdot)$ are constant on the tiles in $w$, and $0 \in \mathbf{R}^{2}$ is the lower left vertex of tile $(0,0)$. Let $\ell=\ell_{2}\left(u_{2}\right)$ be the 0 -line of the 2 -grid $g_{2}\left(u_{2}\right)$. Note that $\ell$ passes through the lower edge of tile $(0,0)$. Let $v$ be the distance along this edge to the point of intersection. Then $0<v<\sec (\pi / 10)$ and $u_{2}=\cos (\pi / 10) v$, (see Figure 5 ).

We will use $\left(a_{i}, b_{i}\right)=\left(\mathbf{m}_{0}(\mathbf{t}), \mathbf{m}_{1}(\mathbf{t})\right)$ to follow $\mathbf{t} \in \ell$ through the tiles. We put $\left(a_{0}, b_{0}\right)=(0,0)$ since $\ell$ 'starts' in the tile $(0,0)$. Now suppose $\left(a_{i}, b_{i}\right)=(a, b)$. As $\ell$ moves northeast, the next tile is either tile $(a+1, b)$ or tile $(a, b+1)$. In the first case, we put $\left(a_{i+1}, b_{i+1}\right)=(a+1, b)$; in the second, we continue to the next tile, $(a+1, b+1)$, and define $\left(a_{i+1}, b_{i+1}\right)=(a+1, b+1)$. A similar procedure defines $\left(a_{i}, b_{i}\right)$ for $i<0$. Note that $\ell$ passes through each tile $\left(a_{i}, b_{i}\right)$, and for each $i \in \mathbf{Z}$, $b_{i+1}=b_{i}+1$ and $a_{i+1}=a_{i}+z_{i}$ where $z_{i}=0$ or $z_{i}=1$. Let $z$ be the sequence with entries $z_{i}$.

Let $\alpha=1-2 \sin (\pi / 10)=\frac{3-\sqrt{5}}{2}$. Viewing the lower edge $e$ of a tile in $w$ as the fundamental domain for a circle, we see that $\ell$ intersects the next copy $e^{\prime}$ of a lower edge of a $w$-tile in a position advanced by $\alpha$. Let $i \subseteq e$ be the subinterval determined by a line parallel to $\ell$ through the upper right vertex of the tile (see Figure 5). In the identification of $e$ with the circle, $i$ is identified with the interval $[\alpha, 1)$. Moreover, $z_{i}=1$ if and only if $\ell$ intersects $i$ (see Figure 5). Since $\mathbf{u}$ is nonsingular, $\ell$ never meets a vertex of $w$, so it contains no endpoints of $[\alpha, 1)$. Thus we have that the sequence $z \in Z_{n}$ for the Sturmian dynamical system with $\alpha=\frac{3-\sqrt{5}}{2}$. Also $v=\sec (\pi / 10) \psi(z)$ and $u_{2}=\psi(z)$.

\footnotetext{
${ }^{3}$ This system is frequently referred to as the Sturmian minimal set, see [6].
} 


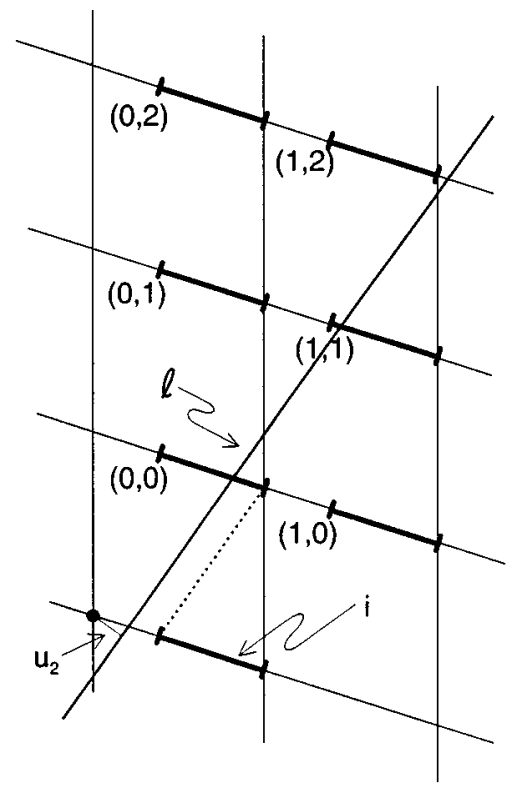

Figure 5. The Sturmian system inside a grid tiling (compare to Figure 3 ).

For $\mathbf{u} \in \mathbf{T}_{0, n}^{5}$, let $N(\mathbf{u})=\left\{\mathbf{m}(\mathbf{s}, \mathbf{u}): \mathbf{s} \in \mathbf{R}^{2}\right\}$ and $\mathbf{N}=\left\{N(\mathbf{u}): \mathbf{u} \in \Sigma\left(\mathbf{T}_{0, n}^{5}\right)\right\} \subseteq$ $\mathbf{Z}^{5}$. Consider the mapping $\mathbf{u} \mapsto N(\mathbf{u}): \Sigma\left(\mathbf{T}_{0, n}^{5}\right) \rightarrow \mathbf{N}$. For $t>0$ let

$$
C_{t}^{c}=\left\{\left(n_{0}, \ldots, n_{4}\right) \in \mathbf{Z}^{5}:\left|n_{i}\right|>t, i=0,1, \ldots, 4\right\} .
$$

Let $\triangle$ denote the symmetric difference operation, and let $\|\cdot\|$ denote Euclidean distance in $\mathbf{T}^{5}$.

Lemma 5.6. The mapping $\mathbf{u} \mapsto N(\mathbf{u})$ is 1:1. The inverse mapping, denoted $\omega: \mathbf{N} \rightarrow \Sigma\left(\mathbf{T}_{0, n}^{5}\right)$, has the property that for all $\epsilon>0$ there exists $t>0$ such that if $N, N^{\prime} \in \mathbf{N}$ satisfy $N \triangle N^{\prime} \subseteq C_{t}^{c}$, then $\left\|\omega(N)-\omega\left(N^{\prime}\right)\right\|<\epsilon$.

Proof. Given $N=N(\mathbf{u}) \in \mathbf{N}$, we have $(p, q,-1, r, s) \in N$ if and only if $\ell$ passes through tile $(p, q)$ in $w(\mathbf{u})$. Putting a lexicographic order on the set of pairs $(p, q)$ which arise as the first two coordinates of $(p, q,-1, r, s)$, we construct a sequence $\left(a_{i}, b_{i}\right)$, which we use to construct $z$. By the previous lemma $u_{2}=\psi(z)$. In a completely analogous way we obtain $u_{4}$ by reading off from $N$ how the line $\ell_{4}\left(u_{4}\right)$ travels through $w$. Since $u_{0}=u_{1}=0$ and $\mathbf{u} \in \mathbf{T}_{0}^{5}$, this determines $\mathbf{u} \in \Sigma\left(\mathbf{T}_{0, n}^{5}\right)$ uniquely.

If $N$ and $N^{\prime}$ agree on $C_{t}$, then the corresponding sequences $c$ and $c^{\prime}$ agree for $-t / 2<i<t / 2$. Given $\epsilon>0$, it follows from the uniform continuity of $\psi$ that if this $R$ is sufficiently large, then $\left|u_{2}-u_{2}^{\prime}\right|=\left|\psi(z)-\psi\left(z^{\prime}\right)\right|<\epsilon$. The same arguments apply to $u_{4}$.

We define $\Sigma\left(X_{n}\right)$ to be the set of all $x \in X_{n}$ such that $0 \in \mathbf{R}^{2}$ is the upper right vertex of a translate of the prototile $\tau_{1}=\left(\ell_{0} \cap \ell_{1}\right)^{*}$ (Figure 6 ). Every such tile is dual to a crossing between a 0 -grid and 1 -grid line in $x$ (i.e., a vertex in $w$ ). 


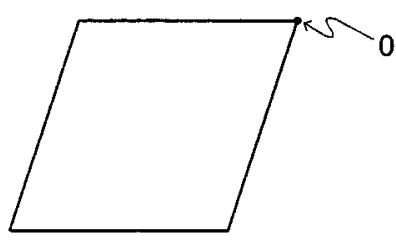

(a)

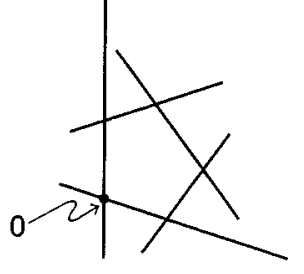

(b)

Figure 6 . The tile (a) $\tau$ in $x \in \Sigma\left(X_{n}\right)$ with its vertex at 0 , and (b) the translation $\sigma$ of $0^{*}$ with its vertex at 0 .

Comment. There is a natural $\mathbf{Z}^{2}$ action $\sigma$ defined on $\Sigma\left(X_{n}\right)$. Let $\mathbf{e}_{1}$ and $\mathbf{e}_{2}$ denote the standard basis vectors for $\mathbf{Z}^{2}$. For $x \in \Sigma\left(X_{n}\right)$ define $\sigma^{\mathbf{e}^{1}} x$ as follows. Let $y$ be the grid tiling dual to $x$ and let $\mathbf{s}$ be the vertex in $y$ dual to the copy of $\tau_{1}$ with a vertex at the origin (it is an intersection of a 0 -grid and a 1 -grid line). Let $\mathbf{s}^{\prime}$ be the next intersection of a 0-grid and a 1-grid line in a positive direction (southeast) along the 1-grid line in $y$. Choose $\mathbf{t}\left(\mathbf{e}_{1}\right) \in \mathbf{R}^{2}$ so that 0 is the upper right vertex of the copy of $\tau_{1}$ in $T^{\mathbf{t}\left(\mathbf{e}_{1}\right)} x$ which is dual to $\mathbf{s}^{\prime}$. We define $\sigma^{\mathbf{e}_{1}} x=T^{\mathbf{t}\left(\mathbf{e}_{1}\right)} x$. We define $\sigma^{\mathbf{e}_{2}}$ in the same way, except that we move up along the 0-grid line from $\mathbf{s}$. One can interpret $\sigma$ as a $\mathbf{Z}^{2}$-symbolic dynamical system as follows. Consider all the different ways the 2, 3 and 4-grid lines cross the tiles in $z$, saying two such pictures are equivalent if (including their boundaries) they have the same dual. Clearly there are only finitely many such equivalence classes. These classes will be the alphabet, and the action $\sigma$ of $\mathbf{Z}^{2}$ will consist of translations along the 0 and 1 -grid lines. It is easy to see that this $\mathbf{Z}^{2}$-action consists of a rotation action on $\mathbf{T}^{4}$, coded by a partition of $\mathbf{T}^{4}$ into polytopes. Clearly the $\mathbf{R}^{2}$ Penrose shift is a (nonconstant) suspension of this $\mathbf{Z}^{2}$-action. The value of the suspension cocycle on the unit basis vectors $\mathbf{e}_{1}, \mathbf{e}_{2} \in \mathbf{Z}^{2}$ depends only on the symbol at time $0 \in \mathbf{Z}^{2}$. It is the length of the "sequence" of tiles dual to the (vertical or horizontal) edge of one equivalence classe. Equivalently, this is the return time to $\Sigma\left(X_{n}\right)$.

Lemma 5.7. For any $x \in X_{n}$ there exist infinitely many $\mathbf{t} \in \mathbf{R}^{2}$ such that $T^{\mathbf{t}} x \in$ $\Sigma\left(X_{n}\right)$. Moreover, at least one such $\mathbf{t}$ satisfies $\|\mathbf{t}\|<8$.

This follows from the fact that the diameter of the patch dual to any of the equivalence classes discussed above is $\leq 8$, and $x(\mathbf{u})$ is made up of a union of such patches.

Given $x \in \Sigma\left(X_{n}\right)$, we define $\mathbf{m}: \mathbf{Z}^{5} \rightarrow q(x)$ inductively by putting $\mathbf{m}(0)=0$ and extending to adjacent vertices using $\mathbf{m}\left(\mathbf{t} \pm \mathbf{v}_{j}\right)=\mathbf{m}(\mathbf{t}) \pm \mathbf{e}_{j}$. That $\mathbf{m}$ is well defined follows from the fact that the sum of $\mathbf{m}$ around the vertices of any tile is zero. Let $N(x)=\{\mathbf{m}(\mathbf{t}): \mathbf{t} \in q(x)\}$.

Suppose $x \in \Sigma\left(X_{n}\right) \subseteq X_{n}$. By the definition of $X_{n}, x=T^{\mathbf{t}\left(\mathbf{u}^{\prime \prime}\right)} y^{*}\left(\mathbf{u}^{\prime \prime}\right)$ for some $\mathbf{u}^{\prime \prime} \in \mathbf{T}_{0, n}^{5}$, so $x$ is geometrically dual to $y\left(\mathbf{u}^{\prime \prime}\right)$. Let $\mathbf{t}$ be the vertex in $y\left(\mathbf{u}^{\prime \prime}\right)$ dual to the tile $\tau$ in $x$ which has its upper right corner at 0 . By duality, $\mathbf{t}$ is at an intersection of a 0 -grid line and a 1-grid line (Figure 6). Letting $\mathbf{u}^{\prime}=\mathbf{u}^{\prime \prime}-W \mathbf{t}$, we have $y\left(\mathbf{u}^{\prime}\right)=y\left(\mathbf{u}^{\prime \prime}-W \mathbf{t}\right)=T^{-\mathbf{t}} y\left(\mathbf{u}^{\prime \prime}\right)$. Thus $y\left(\mathbf{u}^{\prime}\right)$ is also dual to $x$. But the vertex in $y\left(\mathbf{u}^{\prime}\right)$ dual to $\tau$ (which is the intersection of the 0-lines in both the 0-grid and the 1-grid) is at the origin (Figure $6(\mathrm{~b}))$. Let $\sigma$ be the tile in $y\left(\mathbf{u}^{\prime}\right)$ with 0 as a vertex and which is on the positive side of 0 -lines in both the 0 -grid and the 
1-grid (Figure 6(b)). For $\mathbf{r} \in \sigma, \mathbf{m}\left(\mathbf{r}, \mathbf{u}^{\prime}\right)=0$. It follows that $N(x)=N\left(\mathbf{u}^{\prime}\right)$. Thus $N(x) \in \mathbf{N}$ and

$$
\mathbf{u}^{\prime}=w(N(x))
$$

and also $x=y^{*}\left(\mathbf{u}^{\prime}\right)$. Let

$$
\mathbf{u}=K^{-\mathbf{t}\left(\mathbf{u}^{\prime}\right)} \mathbf{u}^{\prime}
$$

By (3) and (6),

$$
\begin{aligned}
x(\mathbf{u})=x\left(K^{-\mathbf{t}\left(\mathbf{u}^{\prime}\right)} \mathbf{u}^{\prime}\right) & =T^{-\mathbf{t}\left(\mathbf{u}^{\prime}\right)} x\left(\mathbf{u}^{\prime}\right) \\
& =y^{*}\left(\mathbf{u}^{\prime}\right) \\
& =x .
\end{aligned}
$$

We define $\varphi: \Sigma\left(X_{n}\right) \rightarrow \mathbf{T}_{0, n}^{5}$ by $\varphi(x)=\mathbf{u}$. By $(7), x(\varphi(x))=x$, which implies $\varphi$ is $1: 1$, and $\varphi(x(\mathbf{u}))=\mathbf{u}$. Then (5), (6) and (2) imply $\varphi(x)=\left(I-\frac{2}{5} W W^{T}\right) \omega(N(x))$. By Lemma 5.6, $\varphi$ is uniformly continuous.

Lemma 5.8. Suppose $x, T^{\mathbf{t}} x \in \Sigma\left(X_{n}\right)$ for some $\mathbf{t} \in \mathbf{R}^{2}$. Then

$$
\varphi\left(T^{\mathbf{t}} x\right)=K^{\mathbf{t}} \varphi(x) \text {. }
$$

Proof. Let $\mathbf{u}=\varphi(x)$ and $\mathbf{u}^{\prime}=\varphi\left(T^{\mathbf{t}} x\right)$, so that by Lemma $5.3, x=T^{-\mathbf{t}} x\left(\mathbf{u}^{\prime}\right)=$ $x\left(K^{-\mathbf{t}} \mathbf{u}^{\prime}\right)$. Then

$$
\begin{aligned}
\varphi(x) & =\varphi\left(x\left(K^{-\mathbf{t}} \mathbf{u}^{\prime}\right)\right) \\
& =K^{-\mathbf{t}} \mathbf{u}^{\prime} \\
& =K^{-\mathbf{t}} \varphi\left(T^{\mathbf{t}} x\right) .
\end{aligned}
$$

Proof of Lemma 5.4. For $x \in X_{n}$, choose $\mathbf{s} \in \mathbf{R}^{2}$ so that $\|\mathbf{s}\| \leq 8$ and $T^{\mathbf{s}} x \in \Sigma\left(X_{n}\right)$, and define

$$
\varphi(x)=K^{-\mathbf{s}} \varphi\left(T^{\mathbf{s}} x\right) .
$$

It follows from Lemma 5.8 that (10) does not depend on the choice of s. Moreover, $\varphi$ is uniformly continuous, since $\left.\varphi\right|_{\Sigma\left(X_{n}\right)}$ is uniformly continuous and $\|\mathbf{s}\|$ is bounded.

Now suppose $x$ is such that $T^{\mathbf{s}} x \in \Sigma\left(X_{n}\right)$ for some $\mathbf{s} \in \mathbf{R}^{2}$. Then $T^{\mathbf{s}-\mathbf{t}}\left(T^{\mathbf{t}} x\right) \in$ $\Sigma\left(X_{n}\right)$, and it follows that

$$
\begin{aligned}
\varphi\left(T^{\mathbf{t}} x\right) & =K^{-(\mathbf{s}-\mathbf{t})} \varphi\left(T^{\mathbf{s}-\mathbf{t}}\left(T^{\mathbf{t}} x\right)\right) \\
& =K^{\mathbf{t}}\left(K^{-\mathbf{s}} \varphi\left(T^{\mathbf{s}}(x)\right)\right) \\
& =K^{\mathbf{t}}(\varphi(x)) .
\end{aligned}
$$

We define the singular Penrose tilings $X_{s}=X \backslash X_{n}$, and call $\mathbf{T}_{0, s}^{5}=\mathbf{T}_{0}^{5} \backslash \mathbf{T}_{0, n}^{5}$ and $Y_{s}=y\left(\mathbf{T}_{0, s}^{5}\right)$ the singular vectors and singular grids respectively. It was shown by de Bruijn [2] that the restriction $\mathbf{u} \in \mathbf{T}_{0}^{5}$ (i.e., not just $\mathbf{u} \in \mathbf{T}^{5}$ ) implies that there can be no 4 -fold crossings in $y(\mathbf{u})$, and moreover, every nonsingular Penrose 5-grid $y(\mathbf{u})$ is of one of the following two types: In Type $A$ all the multiple crossings in $y(\mathbf{u})$ are 3 -fold crossings. There are two kinds of 3-fold crossings: In the first kind, two lines cross with angle $2 \pi / 5$, bisected by the third line. In the second kind lines cross with angle $4 \pi / 5$. All 3 -fold crossings occur along a single grid line. In Type 
$B$ there is a unique 5 -fold crossing in $y(\mathbf{u})$, infinite sequences of 3 -fold crossings along each of the five grid lines in the 5 -fold crossing. There are no other multiple crossings. The following proposition paraphrases a result of de Bruijn:

Proposition 5.9 (de Bruijn [2]). (i) If $x$ is a singular penrose tiling then there exists a singular vector $\mathbf{u}_{0}$, a sequence $\mathbf{u}_{k}$ of nonsingular vectors with $\mathbf{u}_{k} \rightarrow \mathbf{u}_{0}$, and a translation $T^{\mathbf{t}}$ such that

$$
x=T^{\mathbf{t}}\left(\lim _{k \rightarrow \infty} y^{*}\left(\mathbf{u}_{k}\right)\right) .
$$

(ii) Suppose $\mathbf{u}_{0}$ is a singular pentagrid and let $Y^{*}\left(\mathbf{u}_{0}\right)$ denote the the set of limit points of sequences of the form $y^{*}\left(\mathbf{u}_{k}\right)$, where $\mathbf{u}_{k}$ is a sequence of nonsingular vectors with $\mathbf{u}_{k} \rightarrow \mathbf{u}_{0}$. If $\mathbf{u}_{0}$ is Type $A$ then $\operatorname{card}\left(Y^{*}\left(\mathbf{u}_{0}\right)\right)=2$. If $\mathbf{u}_{0}$ is Type $B$ then $\operatorname{card}\left(Y^{*}\left(\mathbf{u}_{0}\right)\right)=10$.

We will need the following refinement of (i).

Proposition 5.10. If $x$ is a singular Penrose tiling then there exists a singular vector $\mathbf{u}(x)$ such that for some sequence $\mathbf{u}_{k}$ of nonsingular vectors with $\mathbf{u}_{k} \rightarrow \mathbf{u}(x)$,

$$
x=\lim _{k \rightarrow \infty} x\left(\mathbf{u}_{k}\right)
$$

In particular, since each $x\left(\mathbf{u}_{k}\right)$ is a nonsingular Penrose tiling, it follows that $X=$ $\overline{X_{n}}$.

Proof. Suppose $x=T^{\mathbf{t}}\left(\lim _{k \rightarrow \infty} y^{*}\left(\mathbf{u}_{k}^{\prime}\right)\right)$ where $\mathbf{u}_{k}^{\prime} \rightarrow \mathbf{u}_{0}^{\prime}$. Then

$$
\begin{aligned}
x & =\lim _{k \rightarrow \infty} y^{*}\left(K^{\mathbf{t}} \mathbf{u}_{k}^{\prime}\right) \\
& =\lim _{k \rightarrow \infty} T^{-\mathbf{t}\left(\mathbf{u}_{k}^{\prime}\right)} x\left(K^{\mathbf{t}} \mathbf{u}_{k}^{\prime}\right) \\
& =\lim _{k \rightarrow \infty} x\left(K^{-\mathbf{t}\left(\mathbf{u}_{k}^{\prime}\right)+\mathbf{t}} \mathbf{u}_{k}^{\prime}\right) .
\end{aligned}
$$

Letting $\mathbf{u}_{k}=K^{-\mathbf{t}\left(\mathbf{u}_{k}^{\prime}\right)+\mathbf{t}} \mathbf{u}_{k}^{\prime}$, it follows that $\mathbf{u}_{k} \rightarrow K^{-\mathbf{t}\left(\mathbf{u}_{0}\right)+\mathbf{t}} \mathbf{u}_{0}^{\prime}$ as $k \rightarrow \infty$, and we put $\mathbf{u}(x)=K^{-\mathbf{t}\left(\mathbf{u}_{0}\right)+\mathbf{t}} \mathbf{u}_{0}^{\prime}$.

By Lemma 5.4 the mapping $\varphi: X_{n} \rightarrow \mathbf{T}_{0}^{5}$ is uniformly continuous, and thus has a unique continuous extension $\varphi$ to $X$. For $x$ singular, (12) implies that

$$
\varphi(x)=\lim _{k \rightarrow \infty} \mathbf{u}_{k}=\mathbf{u}(x) .
$$

Proposition 5.11. The mapping $\varphi: X \rightarrow \mathbf{T}_{0}^{5}$ is a factor mapping.

Proof. It suffices to show that $\varphi$ is onto. Suppose $\mathbf{u}_{0}$ is a singular and $\mathbf{u}_{k} \rightarrow \mathbf{u}_{0}$. Passing to a subsequence if necessary, we assume $x\left(\mathbf{u}_{k}\right) \rightarrow x$. Applying $\varphi$, we have $\mathbf{u}_{0}=\varphi(x)$.

\section{Corollary 5.12.}

$$
\operatorname{card}\left(\varphi^{-1}(\mathbf{u})\right)= \begin{cases}1 & \text { if } \mathbf{u} \text { is nonsingular, } \\ 2 & \text { if } \mathbf{u} \text { is Type A, } \\ 10 & \text { if } \mathbf{u} \text { is Type B. }\end{cases}
$$

This follows from (ii) of Theorem 5.9 and (14).

Proof of Theorem A. This now follows from Lemma 5.4, Proposition 5.11 and Corollary 5.12. 


\section{The geometry of singular Penrose tilings And the topology on $X$}

If $\mathbf{u}_{0}$ is a Type $A$ singular vector, we assume (wolog) that the 3 -fold crossings in $y\left(\mathbf{u}_{0}\right)$ occur along a 3 -grid line $\ell_{3}$ and that the origin is a crossing of the second kind $\left(u_{0}=u_{1}=u_{3}=0\right)$. Any small perturbation $\mathbf{u}$ of $\mathbf{u}_{0}$ in $\Sigma\left(\mathbf{T}_{0, n}^{5}\right)$ moves $\ell_{3}$ above (a positive perturbation) or below (a negative perturbation) the origin, destroying the 3-fold crossings (see Figure 7).

Let $\mathbf{u}_{k} \rightarrow \mathbf{u}_{0}$ where the $\mathbf{u}_{k}$ are nonsingular and the same sign. Then the limit of $x\left(\mathbf{u}_{k}\right)$ exists and depends only on the sign. We denote it by $x^{+}\left(\mathbf{u}_{0}\right)$ or $x^{-}\left(\mathbf{u}_{0}\right)$ respectively. Clearly $\varphi\left(x^{ \pm}\left(\mathbf{u}_{0}\right)\right)=\mathbf{u}_{0}$, so $\varphi^{-1}\left(\mathbf{u}_{0}\right)=\left\{x^{+}\left(\mathbf{u}_{0}\right), x^{-}\left(\mathbf{u}_{0}\right)\right\}$. To construct $x^{+}\left(\mathbf{u}_{0}\right)$ and $x^{-}\left(\mathbf{u}_{0}\right)$, let $p^{\prime}=p \cup\left\{\eta_{1}, \eta_{2}\right\}$ where $\eta_{1}$ and $\eta_{2}$ are the two hexagons shown in Figure 8(b).

By a straightforward extension of Proposition 5.1, $q\left(\mathbf{u}_{0}\right)$ is the vertex set of a unique tiling $x^{\prime}\left(\mathbf{u}_{0}\right)$ in $X_{p^{\prime}}$. The 3 -fold crossings in $y\left(\mathbf{u}_{0}\right)$ are dual to a strip of hexagons $\eta_{1}$ and $\eta_{2}$ parallel to $\ell_{3}$. Away from this strip, $x^{\prime}\left(\mathbf{u}_{0}\right)$ looks like a nonsingular Penrose tiling. To get $x^{+}\left(\mathbf{u}_{0}\right)$ or $x^{-}\left(\mathbf{u}_{0}\right)$, we resolve all hexagons in the same $(+$ or -$)$ direction (see Figure 8$)$. The strip of tiles in $x^{+}\left(\mathbf{u}_{0}\right)$ or $x^{-}\left(\mathbf{u}_{0}\right)$ obtained by resolving of the hexagons is called a Conway worm (see [7]).

The case of Type $B$ is similar but a little more complicated. For $j=0, \ldots, 9$, let $\mathbf{v}_{j}^{\prime}=\left(\operatorname{Re}\left(e^{\pi i j / 5}\right), \operatorname{Im}\left(e^{\pi i j / 5}\right)\right)$, and let $c_{j}$ denote the interior of the positive cone in $\mathbf{R}^{2}$ spanned by $\mathbf{v}_{j}^{\prime}$ and $\mathbf{v}_{j+1}^{\prime}$. Let $F$ be the matrix with $\mathbf{v}_{2}$ and $\mathbf{v}_{4}$ as rows, and consider perturbations $\mathbf{u}_{0}(\mathbf{s})$ of $\mathbf{u}_{0}$, defined by $\mathbf{u}_{0}(\mathbf{s})=\mathbf{u}_{0}+\left(0,0, u_{2},-u_{2}-u_{4}, u_{4}\right)$, where $\left(u_{2}, u_{4}\right)=\mathbf{u}^{\prime}$ and $\mathbf{u}^{\prime}=F \mathbf{s}$ for small $\mathbf{s}$. If the 5 -fold crossing occurs at $\mathbf{b}_{0}$ in $y(\mathbf{u})$, then in $y\left(\mathbf{u}_{0}(\mathbf{s})\right)$ there will be a crossing of a 0 and a 1 -grid line at $\mathbf{b}_{0}$, but the 2 and 4 -grid lines will cross at $\mathbf{b}_{0}+\mathbf{s}$. There will be 3 -fold crossings in a neighborhood of $\mathbf{b}_{0}$ if $\mathbf{s}$ is on the boundary of one of the cones $c_{0}, \ldots, c_{9}$. Fixing $j$, we choose $\mathbf{s}_{k} \in c_{j}$ such that $\mathbf{s}_{k} \rightarrow 0$ and $\mathbf{u}_{0}\left(\mathbf{s}_{k}\right)$ is nonsingular. Then the limit $x^{j}\left(\mathbf{u}_{0}\right)=\lim _{k \rightarrow \infty} x\left(\mathbf{u}_{0}\left(\mathbf{s}_{k}\right)\right)$ exists and depends only on $j$. Let $p^{\prime \prime}$ consist of (i) the tiles in $p$, (ii) the two hexagons $\eta_{1}$ and $\eta_{2}$ and their rotations by multiples of $2 \pi / 5$, and (iii) the regular unit decagon $\delta$. Because $\mathbf{u}_{0}$ is Type $B, q\left(\mathbf{u}_{0}\right)$ is the vertex set for a tiling $x^{\prime \prime}\left(\mathbf{u}_{0}\right) \in X_{p^{\prime \prime}}$, where $\delta$ is dual to the five-fold crossing ( $\delta$ lies at the 'intersection' of five unresolved Conway worms). A choice of $c_{j}$ determines a resolution of this decagon and a resolution of each worm. Figure 9 shows the resolution of a patch around $\delta$ in $x^{0}\left(\mathbf{u}_{0}\right)$, i.e., $\mathbf{s}_{k} \in c_{0}$.

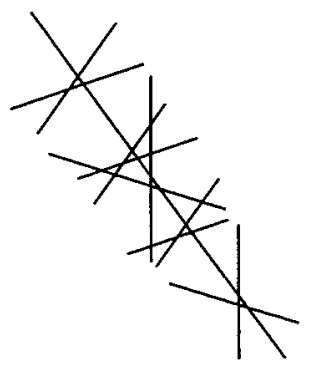

(a)

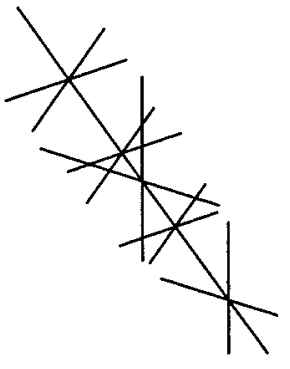

(b)

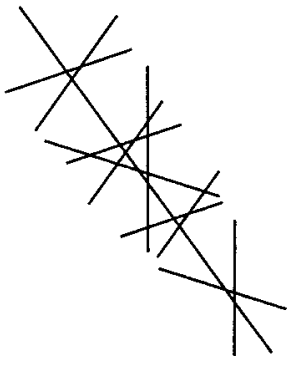

(c)

Figure 7. A Type $A$ singular 5-grid (b), with its positive (a) and negative (c) resolutions. 


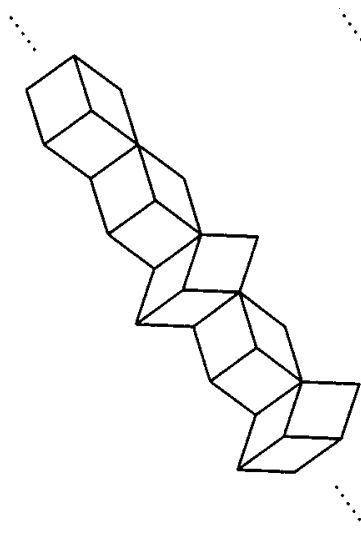

(a)

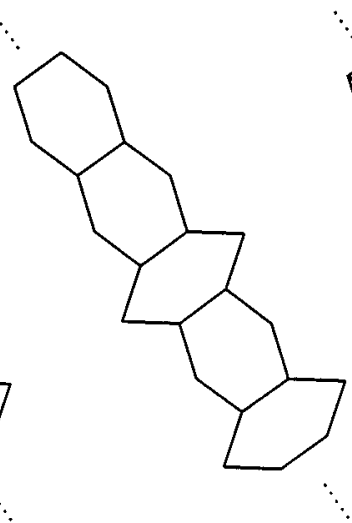

(b)

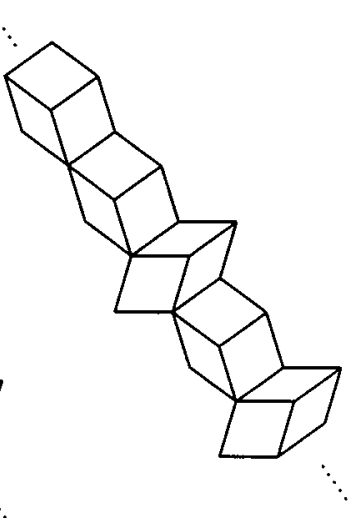

(c)

Figure 8. An unresolved Conway worm (b) made of the hexagons $\eta_{1}$ and $\eta_{2}$, together with its (a) positive and (c) negative resolutions. These pictures are dual to the corresponding pictures in Figure 7 .
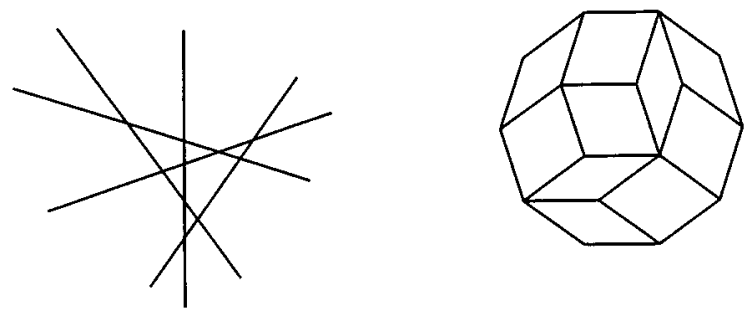

Figure 9. A resolution of the 5-fold crossing in a Type $B$ singular 5 -grid, and its dual the cartwheel; a resolution of the decagon $\delta$.

In fact, since all the resolutions of $x^{\prime \prime}\left(\mathbf{u}_{0}\right)$ may be obtained from $x^{0}\left(\mathbf{u}_{0}\right)$ by a rotation, any two Type $B$ Penrose tilings are congruent. This congruence class is called the cartwheel tiling (see [7]).

Theorem B. Any Penrose tiling $x \in X$ has a neighborhood which is the product of an open set in $\mathbf{R}^{2}$ and a Cantor set.

Proof. We identify $\Sigma\left(\mathbf{T}_{0}^{5}\right)$ with $\mathbf{T}^{2}=\left\{\left(u_{2}, u_{4}\right): 0 \leq u_{2}, u_{4}<1\right\}$ and let $\Sigma_{s}=$ $\Sigma\left(\mathbf{T}_{0}^{5}\right) \backslash \Sigma\left(\mathbf{T}_{0, s}^{5}\right) \subseteq \mathbf{T}^{2}$. First we describe how $\Sigma_{s}$ looks as a subset of $\mathbf{T}^{2}$. Let $\mathbf{u}_{0} \in \mathbf{T}^{2}$ be a Type $B$ singular vector. The points in $\mathbf{T}^{2}$ that correspond to the cones $c_{j}, j=0, \ldots, 9$, are bounded by the lines $\mathbf{u}_{0}+F \mathbf{s}$, where $\mathbf{s} \in \bigcup_{j=0, \ldots, 5}\left\{\mathbf{v}_{j}\right\}^{\perp}$. This set consists of five lines through $\mathbf{u}_{0}$. Three of these (vertical, horizontal and diagonal) have rational slope, and are thus circles in $\mathbf{T}^{2}$. The other two, which have irrational slopes $\frac{-1-\sqrt{5}}{2}$ and $\frac{1-\sqrt{5}}{2}$, wrap densely around $\mathbf{T}^{2}$. We call such configuration of five lines a spider at $\mathbf{u}_{0}$ (see Figure 10(a)).

Since Type $B$ singular vectors are dense in $\mathbf{T}^{2}, \Sigma_{s}$ has spiders at a dense set of points. Any intersection of 2 or more spider legs corresponds to an $n$-fold crossing 
in $y\left(\mathbf{u}_{0}\right)$ with $n>3$. Since there are no 4 -fold crossings, spiders correspond to 5 -fold crossings in $y\left(\mathbf{u}_{0}\right)$ (see Figure 10(b)).

We inductively construct a model for $D=\varphi^{-1}\left(\Sigma\left(\mathbf{T}_{0}^{5}\right)\right)$, replacing each line in the spiders by a pair of lines in $D$. Since there is a spider at $0 \in \mathbf{T}^{2}$, we first remove a vertical and a horizontal circle from $\mathbf{T}^{2}$. Adding a segment to each side, we obtain a closed square which we denote by $C^{\prime}$. We model this as a horizontal square in $\mathbf{R}^{3}$. Now we view $\Sigma_{s}$ as a countable collection of line segments going across $C^{\prime}$ (see Figure 10(b)). In Step 1 we cut along the first segment, separate each half vertically by $1 / 2$, and glue a segment along each side. We denote the result by $C_{1}$. In Step $j$, we cut the $j$ th segment out of $C_{j}$, separate the two halve vertically by $1 / 2^{j}$ and glue segments along both of the edges. Each time we cross a previous cut we obtain a corner (see Figure 11). The resulting union of $\leq 2^{j}$ polygons is denoted $C_{j+1}$. The set $C$, defined to be the set of all limits of sequences $\mathbf{a}_{j} \in C_{j}$, is a Cantor set. Define $\alpha: C \rightarrow \mathbf{T}^{2}$ by $\alpha\left(t_{1}, t_{2}, t_{3}\right)=\left(t_{1}, t_{2}\right)$. Note that $\alpha$ is surjective and $\operatorname{card}\left(\alpha^{-1}(\mathbf{u})\right)$ is either 0,2 or 10 , depending on whether $\mathbf{u}$ is nonsingular (disjoint from the spiders), Type $A$ (in a leg) or Type $B$ (in the center of a spider). Let $C_{0}=\alpha^{-1}\left(\Sigma\left(\mathbf{T}_{0, n}^{5}\right)\right)$ and $D_{0}=\varphi^{-1}\left(\Sigma\left(\mathbf{T}_{0, n}^{5}\right)\right) \subseteq D$. Define a uniformly continuous homeomorphism $\gamma=\left.\varphi^{-1} \circ \alpha\right|_{C_{0}}: C_{0} \rightarrow D_{0}$. This map has a unique extension to a homeomorphism $\gamma: C \rightarrow D$.

Now let $x \in D$ and let $V_{1}=\varphi^{-1}\left(U_{1}\right)$, where $U_{1}$ is a neighborhood of $\varphi(x)$ in $\Sigma\left(\mathbf{T}_{0}^{5}\right)$. Choose $\rho$ sufficiently small that $\left\|\mathbf{t}_{1}\right\|,\left\|\mathbf{t}_{2}\right\|<\rho$ implies $K^{\mathbf{t}_{1}} U_{1} \cap K^{\mathbf{t}_{2}} U_{1}=\phi$ or (equivalently) $T^{\mathbf{t}_{1}} V_{1} \cap T^{\mathbf{t}_{2}} V_{2}=\phi$. Let $U=\bigcup_{\|\mathbf{t}\|<\rho} K^{\mathbf{t}} U_{1}$ and note that $U=$ $U_{1} \times B_{\rho}(0)$, so that $U$ is open. Then $V=\bigcup_{|| \mathbf{t}||<\rho} T^{\mathbf{t}} V_{1}=\varphi^{-1}(U)$ is also open. We claim that $V$ is homeomorphic to $V_{1} \times B_{\rho}(0)$ via the map $t(x, \mathbf{t})=T^{\mathbf{t}} x$. It suffices to show that the inverse is continuous. Given $\epsilon>0$, we choose $\delta>0$ for the uniform equicontinuity of the maps $\left\{T^{\mathbf{t}}:\|\mathbf{t}\| \leq \rho\right\}$. If $x_{1}, x_{2} \in V$ with $h\left(x_{1}, x_{2}\right)<\delta$, then for $i=1,2$ there exists $\left\|\mathbf{t}_{i}\right\|<\rho$ and $z_{i} \in V_{1}$ with $x_{i}=T^{\mathbf{t}_{i}} z_{i}$. Thus $t^{-1}\left(x_{i}\right)=\left(z_{i}, \mathbf{t}_{i}\right)$, and we have $h\left(z_{1}, z_{2}\right)<\epsilon$. Now $\varphi\left(T^{\mathbf{t}_{i}} z_{i}\right)=K^{\mathbf{t}_{i}} \varphi\left(z_{i}\right)$, and $U=U_{1} \times B_{\epsilon}(0)$. Thus since $\varphi$ is continuous, we can ensure that $\left\|\mathbf{t}_{1}-\mathbf{t}_{2}\right\|<\epsilon$ by choosing $\delta$ sufficiently small.

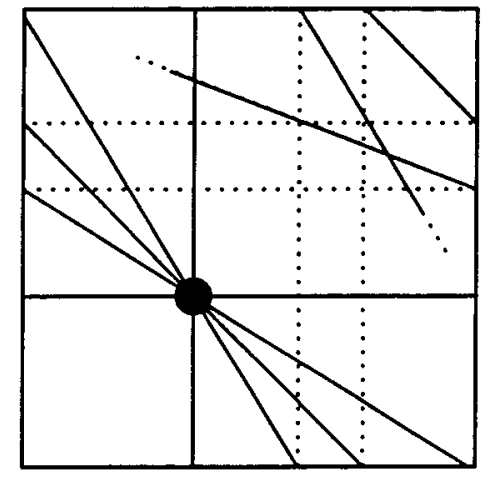

(a)

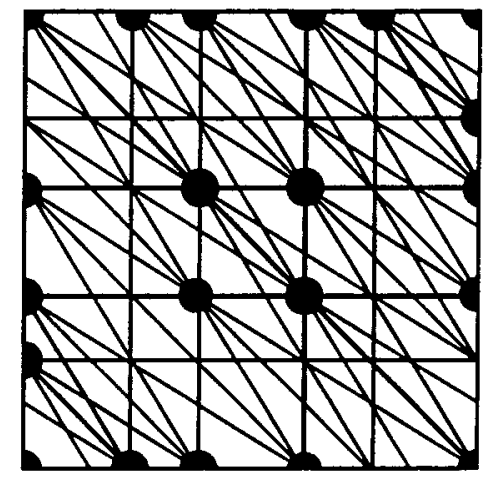

(b)

Figure 10. The torus with (a) one spider, and (b) several spiders. Note that (b) can be viewed as a countable collection of line segments crossing the square. 


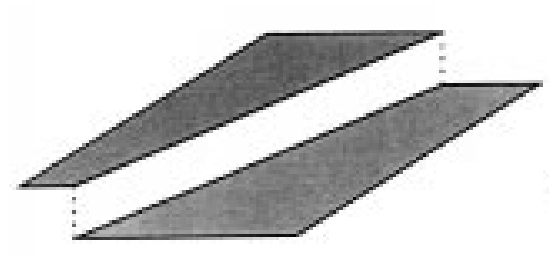

(a)

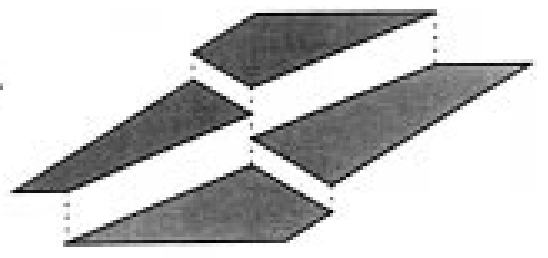

(b)

Figure 11 . The set (a) $C_{1}$, and (b) the set $C_{2}$ showing four corners. Note that two more cuts will eventually go through the same point, so there will ultimately be ten corners there.

For $x \notin D$, we let $\left(u_{0}^{\prime}, u_{1}^{\prime}, \ldots, u_{4}^{\prime}\right)=\varphi(x)$, and replace the set $\Sigma\left(\mathbf{T}_{0}^{5}\right)=\{\mathbf{u} \in$ $\left.\mathbf{T}_{0}^{5}: u_{0}=u_{1}=0\right\}$ with the set $\left\{\mathbf{u} \in \mathbf{T}_{0}^{5}: u_{0}=u_{0}^{\prime}, u_{1}=u_{1}^{\prime}\right\}$.

The author acknowledges helpful conversations with Le Tu Quoc Thang on this subject.

\section{The Penrose inflation}

One of the most intriguing properties of Penrose tilings is inflation. This is most conveniently described in terms of a self-map $Q$ on $X_{\tilde{p}}$. For $x \in X_{\tilde{p}}$, each tile $\tau$ in $x$ is subdivided into patches of smaller scale $\tilde{p}$ tiles (see Figure 12) resulting a new Penrose tiling $\tilde{x}$ on a smaller scale. Then $\tilde{x}$ is scaled up (i.e., inflated) to the original scale. We denote the result by $Q x \in X_{\tilde{p}}$. Clearly $Q$ is a homeomorphism of $X_{\tilde{p}}$, and by conjugating with the mapping $\varepsilon: X_{\tilde{p}} \rightarrow X$, we may assume that $Q$ is a homeomorphism of $X$. Although, as we noted in the Introduction, inflation is reminicient of a substitution rule (see [3], [16], [13]), here we will concentrate on the "hyperbolicity" of $Q$.

The matrix

$$
A=\left(\begin{array}{rrrr}
1 & 1 & 0 & 1 \\
1 & 1 & 1 & 0 \\
-1 & 0 & 0 & -1 \\
0 & -1 & -1 & 0
\end{array}\right)
$$
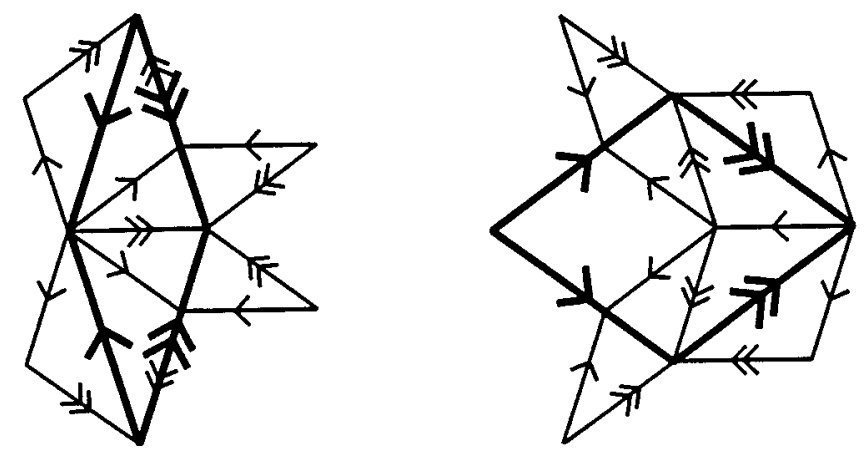

Figure 12. The Penrose inflation. 
is a Lebesgue measure preserving hyperbolic automorphism of $\mathbf{T}^{4}=\mathbf{R}^{4} / \mathbf{Z}^{4}$. The eigenvalues of $A$ are $\frac{1 \pm \sqrt{5}}{2}$, each with multiplicity 2. It is easy to show that $A$ is integrally conjugate to the Cartesian square of the Fibonacci automorphism $\left(\begin{array}{ll}1 & 1 \\ 1 & 0\end{array}\right)$ on $\mathbf{T}^{2}$. Our main result on inflation is the following.

Theorem C. The inflation homeomorphism $Q$ is a nontrivial almost 1:1 extension of the hyperbolic toral automorphism A on $\mathbf{T}^{4}$. The factor map $\varphi$ is the same as the factor map in Theorem A. Moreover, $Q$ preserves the unique T-invariant measure $\mu$ on $X$.

It follows from Theorem $\mathrm{C}$ that $Q$ is topologically mixing and Bernoulli with respect to the unique $T$-invariant measure $\mu$ on $X$. The entropy of $Q$ is $\ln \left(\frac{1+\sqrt{5}}{2}\right)$. However $Q$ is not minimal or uniquely ergodic, since it has infinitely many periodic points.

Proof of Theorem B. It is easy to see that the shift $T$ and the inflation $Q$ satisfy the commutation relation

$$
Q \circ T^{\mathbf{t}}=T^{\lambda \mathbf{t}} \circ Q
$$

where $\lambda=\frac{1+\sqrt{5}}{2}$. Let

$$
B=\left(\begin{array}{lllll}
1 & 1 & 0 & 0 & 1 \\
1 & 1 & 1 & 0 & 0 \\
0 & 1 & 1 & 1 & 0 \\
0 & 0 & 1 & 1 & 1 \\
1 & 0 & 0 & 1 & 1
\end{array}\right)
$$

Then $\mathbf{T}_{0}^{5}$ and $\mathbf{T}_{0, n}^{5}$ are $B$-invariant subsets of $\mathbf{T}^{5}$, and although $\operatorname{det}(B)=3$, the restriction of $B$ to $\mathbf{T}_{0}^{5}$ is invertible. Conjugating by $\pi: \mathbf{T}^{4} \rightarrow \mathbf{T}_{0}^{5}$, we have $\pi \circ A=$ $\left.B\right|_{\mathbf{T}_{0}^{5}} \circ \pi$. A computation shows that the rows of $W^{T}$ are left eigenvectors for $B$, and thus

$$
W^{T} B=\lambda W^{T} .
$$

De Bruijn [2] showed that for all $\mathbf{u} \in \mathbf{T}_{0, n}^{5} Q y^{*}(\mathbf{u})=y^{*}(B \mathbf{u})$, and it follows from this, with (18) and (16) that for $\mathbf{u} \in \mathbf{T}_{0, n}^{5}$,

$$
\begin{aligned}
Q x(\mathbf{u}) & =T^{-\lambda W^{T} \mathbf{u}} Q\left(y^{*}(\mathbf{u})\right) \\
& =T^{-W^{T} B \mathbf{u}} y^{*}(B u) \\
& =x(B \mathbf{u}) .
\end{aligned}
$$

To complete the proof, we conjugate by $\varphi$ and $\pi$, and use continuity to extend to the singular case.

\section{Generalizations}

Here we will briefly mention two ways the situation discussed in this paper can be generalized. First, it is possible to relax the assumption that $\mathbf{u} \in \mathbf{T}_{0}^{5}$ and replace it with the assumption $\mathbf{u} \in \mathbf{T}_{\beta, n}^{5}=\left\{\mathbf{u} \in \mathbf{T}_{n}^{5}: u_{0}+\ldots u_{4}-\beta=0(\bmod 1)\right\}$ for some $\beta \neq 0$. We then construct the corresponding grid tilings $y(\mathbf{u})$, and let $X_{\beta}$ denote the closure of the duals of the nonsingular ones. For $\beta \neq 0$ this yields tilings that are not congruent (or even locally isomorphic) to Penrose tilings (Figure 13). 
It is easy to see that the shift $T$ on each $X_{\beta}$ is an almost automorpic extension of the (same) Kronecker action $K$, and is thus metrically isomorphic to the Penrose shift. However, one can show that for Lebesgue a.e. $\beta$, there is a singular grid tiling with a four-fold crossing. It follows that 8 belongs to the thickness spectrum and thus the corresponding shift is not topologically conjugate to the Penrose shift (although they are metrically isomorphic).

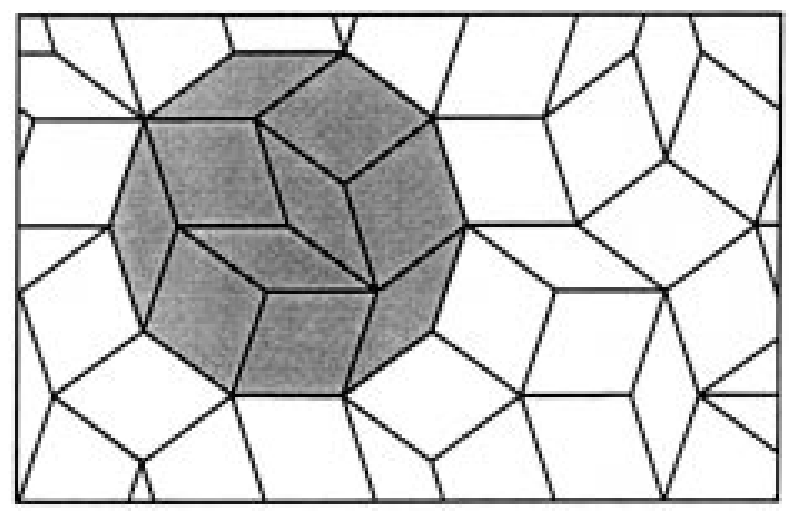

Figure 13. A generalized Penrose tiling for $\beta=1 / 2$. It is easy to see that the shaded patch cannot be decorated with arrows to satisfy the Penrose matching condition, so this patch does not occur in any Penrose tiling.

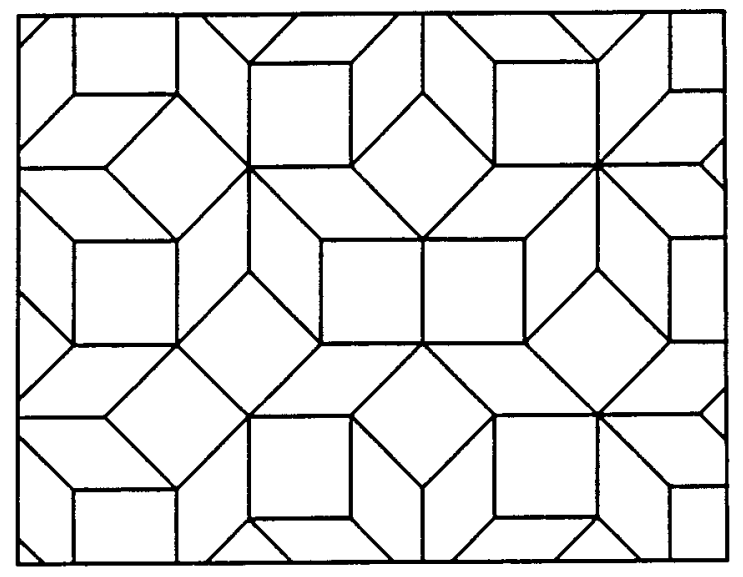

Figure 14. Part of a generalized Penrose tiling for $r=2^{3}$.

In a second generalization, one replaces the 5 th roots of unity with the $r$ th roots of unity for $r \geq 6$. Following Niizeki [10], we let $d=r$ if $r$ is odd and $d=r / 2$ if $r$ is even. For $\mathbf{u} \in \mathbf{T}^{d}$, a grid tiling $y(\mathbf{u})$ consists of $d$ grids perpendicular to the first $d r$ th roots of unity. If such a tiling is nonsingular, its dual is some $x \in X_{p}$, where $p$ is the collection of convex hulls of pairs of the first $d r$ th roots of unity $(\operatorname{card}(p)=d(d-1) / 2)$. Let $X$ denote the closure of all such nonsingular grids. 
As in the case above (where the parameter $\beta$ separates different minimal sets or local isomorphism classes), one can show there is an $a=d-\phi(r)$ parameter family of minimal sets (where $\phi$ denotes the Euler $\phi$-function). Restricted to any such minimal set, the shift $T$ in this case is almost auomorphic with $C_{T}=\mathbf{Z}\left[e^{2 \pi i / r}\right]$. It is interesting to note that $r=2^{t}$ implies $a=0$ (see [10]), so $T$ is minimal on $X$. The smallest such case $r=8, d=4$ (Figure 14) gives the well known Aman tiling (see [7]).

\section{REFERENCES}

1. Arnold, V. I., Huygens and Barrow, Newton and Hooke: pioneers in mathematical analysis and catastrophe theory from evolvents to quasicrystals, Birkhauser Verlag (1990). MR 91h:01014

2. de Bruijn, N. G., Algebraic theory of Penrose's non-periodic tilings of the plane I \& II, Kon. Nederl. Akad. Wetensch. A84, (1981), 39-66. MR 82e:05055

3. Cahn, J. and J. Taylor, An introduction to quasicrystals, The Legacy of Sonya Kovalevskaya, Contemporary Mathematics 64, (1987), 265-286. MR 89a:82032

4. Connes, A., Noncommutative Geometry, Academic Press, (1994). MR 95j:46063

5. Gottschalk, W., Orbit-closure decompositions and almost periodic properties, Bull. Amer. Math. Soc. 50, (1944), 915-919. MR 6:165a

6. Gottschalk, W. and G. Hedlund, Topological Dynamics, Colloquium Publication XXXVI, (1955), Amer. Math. Soc. MR 17:650e

7. Grünbaum, B., and G. C. Shephard, Tilings and Patterns, W. H. Freeman and Company, (1987). MR 88k:52018

8. Ireland, K., and M. Rosen, A Classical Introduction to Modern Number Theory, Springer, 1982. MR 83g: 12001

9. Mozes, S., Tilings, substitution systems and dynamical systems generated by them, $J$. d'Analyse Math. 53, (1989), 139-186. MR 91h:58038

10. Niizeki, K., A classification of two-dimensional quasi-periodic tilings obtained with the grid method, J. Phys. A 21, (1988), 3333-3345. MR 90e:82073

11. Penrose, R., The role of aesthetics in pure and applied mathematical research, Bull. Inst. Math. Appl. 10, (1974), 266-271.

12. Radin, C., and M. Wolff, Space tilings and local isomorphism, Geometria Dedicata 42, (1992), 355-360. MR 93c:52019

13. Robinson, E. A., The dynamical theory of tilings and quasicrystallography, Ergodic Theory of $\mathbb{Z}^{d}$-actions, London Math. Society Lecture Note Series 228, Eds., M. Pollicot and K. Schmidt, Cambridge University Press, 451-473 (1996).

14. Robinson, E. A., The point spectra of quasilattices, in preparation.

15. Rudolph, D. J., Markov tilings of $\mathbf{R}^{n}$ and representations of $\mathbf{R}^{n}$ actions, Contemporary Mathematics 94, (1989) 271-290. MR 91b:28016

16. Solomyak, B. , Dynamics of self-similar tilings, to appear in Ergodic Theory and Dynamical Systems.

17. Thurston, W., Groups, tilings, and finite state automata, AMS Colloquium Lecture Notes, (1989).

18. Veech, W., Topological Dynamics, Bull. Amer. Math. Soc. 83, (1970), 775-830. MR 57:7558

19. Walters, P., Introduction to Ergodic Theory, Springer Verlag, 1981. MR 84e:28017

Department of Mathematics, The George Washington University, Washington, D.C. 20052

E-mail address: robinson@math.gwu.edu 